\title{
HDAC-Linked "Proliferative" miRNA Expression Pattern in Pancreatic Neuroendocrine Tumors
}

\author{
Eckhard Klieser ${ }^{1, \dagger}{ }^{,}$Romana Urbas ${ }^{1, \dagger}$, Stefan Swierczynski ${ }^{2, \dagger}$, Stefan Stättner ${ }^{3}$, \\ Florian Primavesi ${ }^{3}$, Tarkan Jäger ${ }^{2}$ (D), Christian Mayr ${ }^{4,5}$, Tobias Kiesslich ${ }^{4,5}$, Pietro Di Fazio ${ }^{6}$, \\ Katharina Helm ${ }^{1,5,+}$ and Daniel Neureiter $1, t, *$ \\ 1 Institute of Pathology, Cancer Cluster Salzburg, Paracelsus Medical University/Salzburger \\ Landeskliniken (PMU/SALK), Muellner Hauptstrasse 48, 5020 Salzburg, Austria; e.klieser@salk.at (E.K.); \\ r.urbas@salk.at (R.U.); katharina.helm@pmu.ac.at (K.H.) \\ 2 Department of Surgery, Paracelsus Medical University/Salzburger Landeskliniken (PMU/SALK), \\ Muellner Hauptstrasse 48, 5020 Salzburg, Austria; stefan.swierczynski@gmail.com (S.S.); \\ ta.jaeger@salk.at (T.J.) \\ 3 Department of Visceral-, Transplant- and Thoracic Surgery, Medical University of Innsbruck, \\ 6020 Innsbruck, Austria; stefan.staettner@tirol-kliniken.at (S.S.); florian.primavesi@tirol-kliniken.at (F.P.) \\ 4 Department of Internal Medicine I, Paracelsus Medical University/Salzburger \\ Landeskliniken (PMU/SALK), Muellner Hauptstrasse 48, 5020 Salzburg, Austria; \\ christian.mayr@pmu.ac.at (C.M.); t.kiesslich@salk.at (T.K.) \\ 5 Laboratory for Tumor Biology and Experimental Therapies, Institute of Physiology and Pathophysiology, \\ Paracelsus Medical University (PMU), Strubergasse 22, 5020 Salzburg, Austria \\ 6 Department of Visceral, Thoracic and Vascular Surgery, Philipps University of Marburg, Baldingerstrasse \\ 35043 Marburg, Germany; difazio@med.uni-marburg.de \\ * Correspondence: d.neureiter@salk.at; Tel.: +43-(0)57-255-29027 \\ + These authors contributed equally to this work.
}

Received: 31 July 2018; Accepted: 11 September 2018; Published: 15 September 2018

Abstract: Epigenetic factors are essentially involved in carcinogenesis, tumor promotion, and chemoresistance. Two epigenetic key players are miRNAs and histone deacetylases (HDACs). As previously shown by own theoretical databank analysis, the crosstalk between miRNAs and HDACs is relevant in different human chronic diseases and cancerogenic pathways. We aimed to investigate a potential connection between the expression of a well-defined subset of "proliferationassociated" miRNAs and the expression of HDACs as well as clinical parameters in pancreatic neuroendocrine tumors (pNETs). Materials and Methods: Expression levels of miRNA132-3p, miRNA145-5p, miRNA183-5p, miRNA34a-5p, and miRNA449a in 57 pNETs resected between 1997 and 2015 were measured and linked to the immunohistochemical expression pattern of members of the four HDAC classes on human tissue microarrays. All pNET cases were clinically and pathologically characterized according to published guidelines. Correlation analysis revealed a significant association between expression of specific miRNAs and two members of the HDAC family (HDAC3 and HDAC4). Additionally, a linkage between miRNA expression and clinico-pathological parameters like grading, TNM-staging, and hormone activity was found. Moreover, overall and disease-free survival is statistically correlated with the expression of the investigated miRNAs. Overall, we demonstrated that specific miRNAs could be linked to HDAC expression in pNETs. Especially miRNA449a (associated with HDAC3/4) seems to play an important role in pNET proliferation and could be a potential prognostic factor for poor survival. These first data could help, to improve our knowledge of the complex interactions of the epigenetic drivers in pNETs for further therapeutic approaches.

Keywords: epigenetics; miRNA; histone deacetylases; pancreatic neuroendocrine tumor 


\section{Introduction}

Pancreatic neuroendocrine tumors ( $\mathrm{pNETs}$ ) are a rare group of neoplasms originating from the pancreas that exhibits endocrine phenotypes. They can be divided into functioning and non-functioning pNETs, with the latter representing the more common group. Functioning pNETs are characterized by specific hormonal hyper secretion and can be classified in insulinomas (45\%), gastrinomas (20\%), glucagonomas (13\%), vasoactive intestinal peptide (VIP)-omas (10\%), and somatostatinomas (5\%) [1-3]. Incidence rates currently lie at 1-4 per 100,000 population per year but are increasing exponentially, mostly due to improved imaging techniques and concurrent detection rate [4]. Surgical resection is the primary therapeutic option for patients with pNET. Even though there are alternative and multidisciplinary therapeutic approaches, they have failed to provide prolonged survival benefit so far [5]. Due to this and the fact that surgery is not always applicable, there is an urgent need for new therapeutic targets and treatment options.

Aberrant epigenetic regulation of gene function is strongly linked to cancer initiation and progression [6,7]. Epigenetic malfunctions have been found to cause altered gene expression and epithelial to mesenchymal transmission (EMT). Epigenetic regulations can be mediated by DNA methylation, histone modifications or microRNAs (miRNAs) [8,9]. Especially, aberrations in histone modifying mechanisms such as histone de-/acetylation seem to play a significant role in cancer [10]. In the context of pNETs, histone deacetylation (and consequently silencing) of tumor suppressor genes may contribute to the formation of tumors arising from the exocrine and endocrine pancreas [11]. These deacetylation processes are regulated by a group of enzymes called histone deacetylases (HDACs), which can be allocated to four classes: Class I, IIA, IIB, III, and IV. Overexpression of HDACs has been reported in several cancer types including pNETs, making these enzymes potential therapeutic targets [12]. Another group of epigenetic regulating factors involved in cancerogenesis are miRNAs, which can function as both oncogenes and tumor suppressor genes (TSGs) [13-18]. MicroRNAs are single-stranded small non-coding RNAs with 18-25 nucleotides in length. Beyond their involvement in a diverse range of biological processes, miRNAs (oncogenic) are linked to several pro-tumorigenic processes, such as apoptosis resistance, cell proliferation, chemoresistance and EMT, dependent on the function of their respective target mRNA. Interestingly, previous studies demonstrated that some miRNAs directly interact with HDAC-mediated mechanisms by modulating genes encoding for particular HDACs $[19,20]$. Further research regarding these epigenetic mechanisms could lead to the development of improved treatment strategies by a combinatory approach targeting HDACs and specific miRNAs involved in cancer development and progression [21]. Based on these promising findings by other working groups regarding expression of specific miRNAs and HDAC expression, and based on the urgent requirement for new therapeutic strategies and/or targets in pNETs, we, therefore, aimed to investigate the expression levels of a well-defined subset of "proliferation-associated" miRNAs (miRNA132-3p, miRNA145-5p, miRNA183-5p, miRNA34a-5p, and miRNA449a) and their correlation with immunohistochemical expression patterns of members of the four HDAC classes. Moreover, we analyzed the association between these expression patterns and clinico-pathological parameters in pNETs.

\section{Results}

\subsection{Clinical Characteristics of pNET Patients}

As summarized in Table 1, 57 cases $(n=32(56.1 \%)$ male and $n=25(43.9 \%)$ female with a mean age of $60.9 \pm 14.4$ years) with pNET $(n=27(47.4 \%)$ immunohistochemically endocrine positive) were included in the study. According to the WHO the pNETs were graded as NET G1 $(n=32(56.1 \%))$, NET G2 $(n=17(29.8 \%))$, and NEC (neuroendocrine carcinoma) G3 $(n=8(14.0 \%))$ with a mean Ki-67 associated proliferative and $\mathrm{H} \& \mathrm{E} / \mathrm{pHH} 3$-associated mitotic activity of $10.8 \%$ and 12.8/15.0 per 10 high power fields. Related to the TNM, the pNET tumors had a mean size of $2.69 \pm 2.34 \mathrm{~cm}$, mainly located in the head of the pancreas $(n=25(44.6 \%))$ and presented mainly in TNM-stage T1 in $n=24(42.1 \%)$ 
with a locoregional lymph node and distant metastasis rate in $n=19(33.3 \%)$ and in $n=11$ cases $(19.3 \%)$, respectively. Finally, significant differences were observed for TNM, R status and events of overall survival (OS) and disease-free survival (DFS) inside the classical clinical parameters (using $\chi^{2}$ test, $\left.{ }^{*} p<0.05\right)$.

Table 1. Patient characteristics of pancreatic neuroendocrine tumors (pNET).

\begin{tabular}{|c|c|c|}
\hline Parameters & Cases & $p$ Value $^{\text {a }}$ \\
\hline \multicolumn{3}{|c|}{ Gender, $n(\%)$} \\
\hline Male & $32(56.1 \%)$ & \multirow{2}{*}{0.354} \\
\hline Female & $25(43.9 \%)$ & \\
\hline Age (mean \pm SD) & $60.9 \pm 14.4$ & n.a. \\
\hline Size $($ mean $(\mathrm{cm}) \pm \mathrm{SD})$ & $2.69 \pm 2.34$ & n.a. \\
\hline \multicolumn{3}{|c|}{ Localization, $n(\%)^{\mathrm{b}}$} \\
\hline Head & $25(44.6)$ & \multirow{3}{*}{0.067} \\
\hline Corpus & $11(19.6)$ & \\
\hline Cauda & $20(35.7)$ & \\
\hline \multicolumn{3}{|c|}{$\mathrm{TNM}, n^{\mathrm{c}}$} \\
\hline $\mathrm{T} 1 / 2 / 3 / 4$ & $24 / 13 / 17 / 3$ & ** 0.001 \\
\hline N0/1 & $38 / 19$ & *0.012 \\
\hline $\mathrm{M} 0 / 1$ & $46 / 11$ & $* * 0.000$ \\
\hline \multicolumn{3}{|c|}{ Grading, $n(\%)$} \\
\hline G1 & $32(56.1)$ & \multirow{3}{*}{ n.a. } \\
\hline G2 & $17(29.8)$ & \\
\hline G3 & $8(14.0)$ & \\
\hline \multicolumn{3}{|c|}{ R status, $n(\%)$} \\
\hline R0 & $51(89.5)$ & \multirow{3}{*}{$* * 0.000$} \\
\hline $\mathrm{R} 1$ & $5(8.8)$ & \\
\hline $\mathrm{R} 2$ & $1(1.8)$ & \\
\hline \multicolumn{3}{|c|}{ OS, $n(\%)$} \\
\hline Yes & $47(82.5)$ & \multirow{2}{*}{$* * 0.000$} \\
\hline No & $10(17.5)$ & \\
\hline \multicolumn{3}{|c|}{ DFS, $n(\%)$} \\
\hline Yes & $34(73.9)$ & \multirow{2}{*}{ ** 0.001} \\
\hline No & $12(26.1)$ & \\
\hline \multicolumn{3}{|c|}{ Proliferation/Mitosis } \\
\hline$H \& E^{d}$ & $12.8 \pm 27.1$ & \multirow{3}{*}{ n.a. } \\
\hline $\mathrm{pHH} 3^{\mathrm{e}}$ & $15.0 \pm 31.4$ & \\
\hline Ki-67 (\%) ${ }^{f}$ & $10.8 \pm 21.3$ & \\
\hline \multicolumn{3}{|c|}{ Hormone activity $n(\%) \mathrm{g}$} \\
\hline No & $30(52.6)$ & \multirow{2}{*}{0.691} \\
\hline Yes & $27(47.4)$ & \\
\hline
\end{tabular}

${ }^{a} \chi^{2}$ test; ${ }^{b}$ Reduced case numbers due to unknown data; ${ }^{c}$ according to current TNM (7th edition (2010));

${ }^{\mathrm{d}} \mathrm{H \& E}$-associated mitotic activity per 10 high power fields; ${ }^{\mathrm{e}} \mathrm{pHH}$-associated mitotic activity per 10 high power fields; ${ }^{\mathrm{f}} \mathrm{Ki}-67$-associated proliferation index (\% per 2000 tumor cells); ${ }^{\mathrm{g}}$ measured by immunohistochemistry; n.a., not applicable; SD, standard deviation.

\subsection{Expression of miRNA in $p N E T s$}

As shown in Figure 1, the miRNA expression analysis of the pNET samples with RT-PCR revealed that miRNA145-5p had the overall highest (significant difference, $p<0.001$, ANOVA, Bonferroni) and miRNA449a, the overall lowest expression levels, respectively (expression levels in descending order: $145-5 p, 34 a-5 p, 132-3 p, 183-5 p, 449 a)$. For further analysis, the expression levels for each individual miRNA were separated into "low" and "high", according to the mean expression score. 
The Youden-index was applied for calculating a significant cut-off-value for each miRNA, based on the OS and DFS rate (as listed in Table 2).

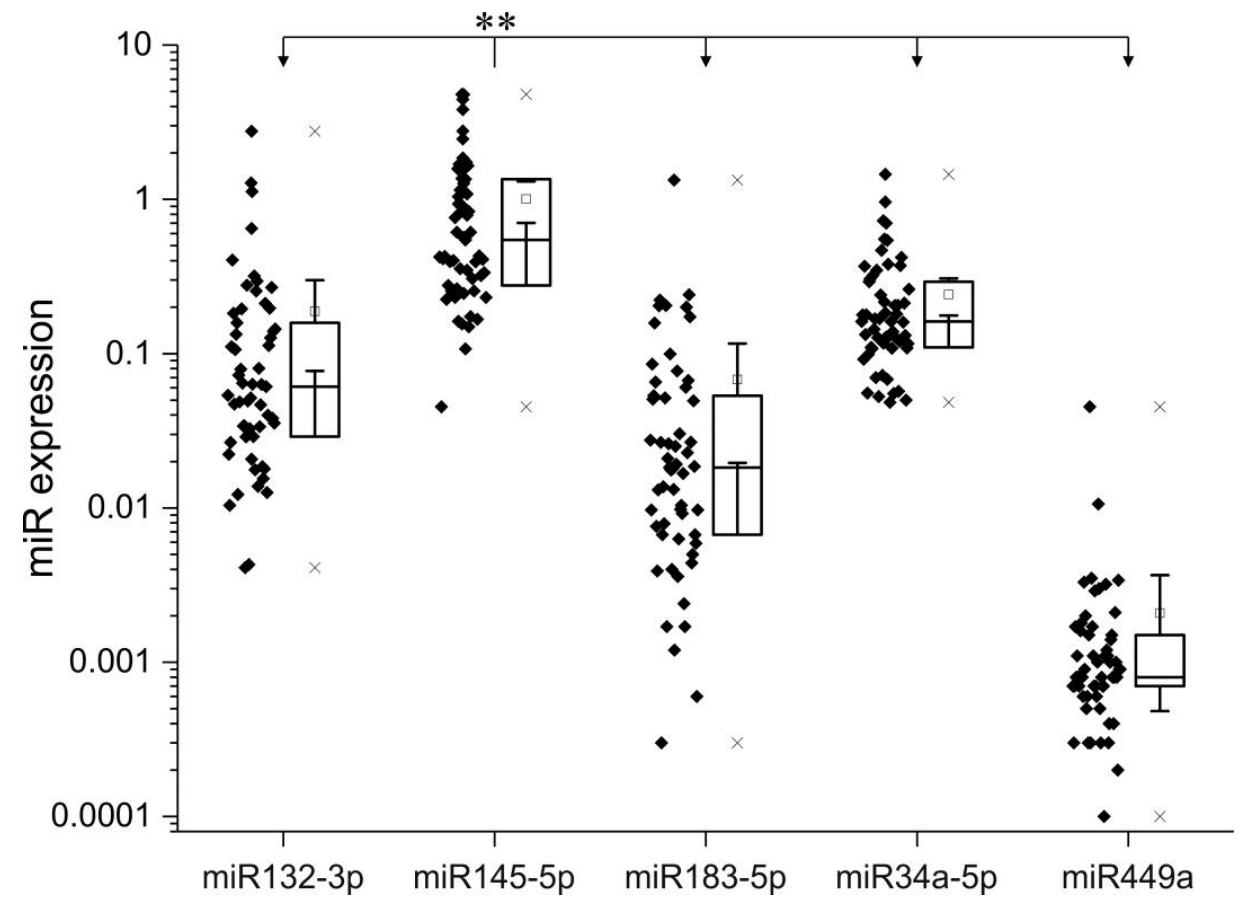

Figure 1. Evaluated expression of miRNA 132-3p, 145-5p, 183-5p, 34a-5p, 449a in the pancreatic neuroendocrine tumors ( $\mathrm{pNET}$ ) patient collective. Box plots showing the expression of individual miRNAs related to housekeeping genes. ${ }^{* *} p<0.01$ (ANOVA, Bonferroni).

Table 2. Cut-off-values based on overall- and disease-free-survival.

\begin{tabular}{cccc}
\hline \multirow{2}{*}{ miRNA- } & \multirow{2}{*}{ Mean \pm SD } & \multicolumn{2}{c}{ Cut-Off-Value (ROC, Youden-Index) } \\
\cline { 3 - 4 } & & Overall-Survival & Disease-Free-Survival \\
\hline $132-3 p$ & $0.18826 \pm 0.41903$ & 0.26125 & 0.06320 \\
$145-5 p$ & $1.0057 \pm 1.14076$ & 0.58855 & 0.39820 \\
$183-5 p$ & $0.06794 \pm 0.18228$ & 0.05250 & 0.05250 \\
$34 a-5 p$ & $0.24186 \pm 0.24647$ & 0.08215 & 0.29910 \\
$449 a$ & $0.00207 \pm 0.00600$ & 0.00310 & 0.00265 \\
\hline
\end{tabular}

\subsection{Correlation Analysis of miRNA and HDAC Expression}

The correlation analysis between miRNA expression and HDAC expression patterns revealed that miRNA449a is significantly positively correlated with relative expression of HDAC3 (nuc) $\left(+^{*}(0.310)\right)$ and HDAC4(cyt) $\left(+^{*}(0.302)\right)$. Representative images of H\&E morphology and HDAC3/4 immunohistochemistry in correlation with and miRNA449a stainings are shown in Supplementary Figure S2. Interestingly, we observed (by trend) higher expression levels of the remaining HDACs in our pNET samples (Supplementary Table S2).

\subsection{Association of miRNA Expression with Grading and Mitotic/Proliferative Status}

All pNET tumor samples were classified according to the TNM-associated WHO-grading system (G1-G3), and their different miRNA expression levels were compared (see Figure 2 and Supplementary Figure S1). Furthermore, low-grade (G1 and G2) cases were grouped, and their miRNA expression level was compared to high-grade (G3). As illustrated in Figure 2, significant differences for miRNA449a between low- and high-graded tumors were observed, whereas remaining miRNAs showed heterogeneous expression patterns (Supplementary Figure S1). The miRNA145-5p, 
miRNA183-5p, and miRNA449a were upregulated in high-grade compared to low-grade pNETs, whereas miRNA132-3p was downregulated in high-graded pNETs compared to low-graded tumors. Of note, the expression of miRNA34a-5p remains stable in pNETs independent of the tumor grading. Interestingly, correlation analysis of miRNA expression and $\mathrm{H} \& \mathrm{E}$ associated mitotic and Ki-67 associated proliferative activity revealed (highly) significant associations for miRNA449a (Figure 2A): H\&E mitoses (absolute $+^{* *}(0.446) /$ relative $\left.+{ }^{* *}(0.366)\right)$, PHH3 (absolute $+^{*}(0.310) /$ relative $+^{*}(0.283)$ ), Ki-67 proliferative activity (absolute $+{ }^{* *}(0.540) /$ relative $\left.+{ }^{*}(0.330)\right)$.

\begin{tabular}{|c|c|c|c|}
\hline \multirow{2}{*}{ A } & \multirow[b]{2}{*}{ ki-67 } & \multicolumn{2}{|c|}{ Mitoses } \\
\hline & & $\mathrm{HE}$ & $\mathrm{PHH} 3$ \\
\hline \multirow{2}{*}{ miR132-3p } & -0.138 & -0.119 & -0.135 \\
\hline & 0.310 & 0.379 & 0.320 \\
\hline \multirow{2}{*}{ miR145-5p } & 0.052 & -0.015 & -0.051 \\
\hline & 0.704 & 0.912 & 0.707 \\
\hline \multirow{2}{*}{ miR183-5p } & -0.049 & -0.055 & -0.053 \\
\hline & 0.718 & 0.683 & 0.698 \\
\hline \multirow{2}{*}{ miR34a-5p } & -0.132 & -0.158 & -0.165 \\
\hline & 0.332 & 0.240 & 0.224 \\
\hline \multirow{2}{*}{ miR449a } & 0.540 & 0.446 & 0.310 \\
\hline & 0.000 & 0.001 & 0.020 \\
\hline
\end{tabular}

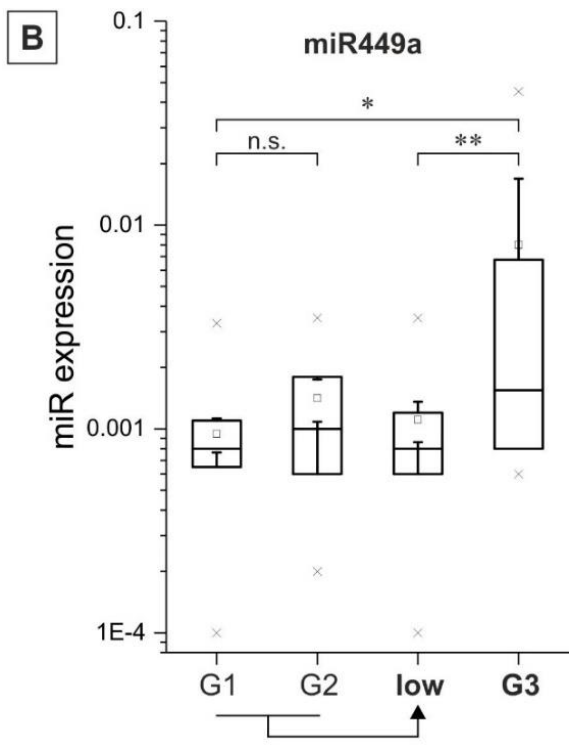

Figure 2. (A) Correlation analysis microRNAs (miRNA) expression and markers of proliferation and mitosis. The upper value stands for the Pearson correlation coefficient (absolute values), and the lower value is the related $p$ value; significant differences are highlighted (black rectangles); (B) Quantitative analysis of pNET-miRNA expression versus grading. miRNA expression is plotted via box plots versus grading of pNET cases. Additionally, the expression levels were summarized for G1 and G2 pNET cases. ${ }^{*} p<0.05,{ }^{* *} p<0.01$ ( $t$-Test and ANOVA).

\section{5. miRNA Expression and Clinicopathological Characteristics}

Next, we compared the absolute and relative miRNA expression with respect to clinico-pathological characteristics. If applicable, the relative miRNA expression levels were classified into low and high according to the mean expression score. Exemplary results are shown in Figure 3. We found that the expression of miRNA183-5p was highly significantly associated with the tumor size (low /high, $+{ }^{* *}(0.433)$, Pearson, data not shown). The Union International contre le cancer (UICC) status (categorized in 1-4) is correlated with higher miRNA183-5p-expression in an increasing manner (highly significant differences between groups $1-4{ }^{* *}(0.000), 2-4{ }^{* *}(0.001), 3-4{ }^{* *}(0.002)$; ANOVA, Bonferroni). In addition, we found a significant group difference between ENET status 1 and 4 $\left({ }^{*}(0.040) ;\right.$ ANOVA, Bonferroni) and the expression of miRNA183-5p (data not shown). No significant differences were found regarding T-status (UICC and ENET) categorized into low and high (data not shown). Lymph node infiltration $(\mathrm{pN})$ is linked to higher expression of miRNA449a (equal variances assumed *(0.047), no homogeneity of variances (Levene) $(0.171)$; $t$-test). Invasion of lymphatic vessels $(\mathrm{L})$ is significantly linked to lower expression of miRNA145-5p $\left({ }^{*}(0.024), t\right.$-test $)$ but there are no significant differences related to podoplanin levels (data not shown). Analysis of the expression of our miRNAs in relation to distant metastasis $(\mathrm{pM})$ did not reveal any significant differences. However, we found that pNET cases with vascular invasion (V) tend to have higher expression levels of miRNA-132-3p $\left({ }^{*}(0.045)\right.$ no homogeneity of variances (Levene) $\left.(0.460)\right)$. The cell surface antigen CD34 was significantly coupled with increased expression levels of miRNA132-3p 
$\left({ }^{*}(0.019), t\right.$-test, data not shown). Regarding the resection margins status R0-R1 (there was only one pNET case with macroscopic residual tumor (R2). Therefore, a $t$-test was calculated between the groups R0 and R1) group differences were found for the expression levels of miRNA132-3p, $34-5 \mathrm{p}$ and $449 \mathrm{a}\left({ }^{*}(0.017),{ }^{* *}(0.000),{ }^{* *}(0.002)\right.$; no homogeneity of variances (Levene) $(0.440,0.186$, $0.382) ; t$-test, data not shown). pNET cases with hormone activity are by trend linked with higher expression of all miRNAs except miRNA132-3p. We then performed a correlation analysis between miRNA expression and the secretion of the hormones Somatostatin, Glucagon, Insulin, Serotonin, Gastrin, Calcitonin, and VIP. Somatostatin secretion was positively correlated with the expression of miRNA132-3p (relative $-{ }^{*}(0.266)$ ) and miRNA34a-5p (relative $-{ }^{*}(0.272)$ ). Levels of serotonin were positively correlated with miRNA145-5p- (absolute $+^{* *}(0.409)$, relative $\left.+^{*}(0.269)\right)$, miRNA183-5p(absolute $+^{* *}(0.944)$, relative $\left.+^{* *}(0.389)\right)$, miRNA34a-5p- (relative $\left.+^{*}(0.304)\right)$ levels. Moreover, we found a positive correlation between Gastrin secretion and expression of miRNA34a-5p $\left(+^{* *}(0.375)\right)$ (data not shown). The expression of the Somatostatin-receptor type 2 (SSTR2) can be categorized into four groups based on the expression levels $(0,1,2,3)$. According to ANOVA and Bonferroni analysis, there are highly significant differences regarding miRNA183-5p and SSTR2 expression $(0-1 * *(0.001)$, $1-2 *(0.002), 1-3 * *(0.000))$. Interestingly, SSTR2 status 1 with low expression levels is linked to the highest expression of miRNA183-5p.
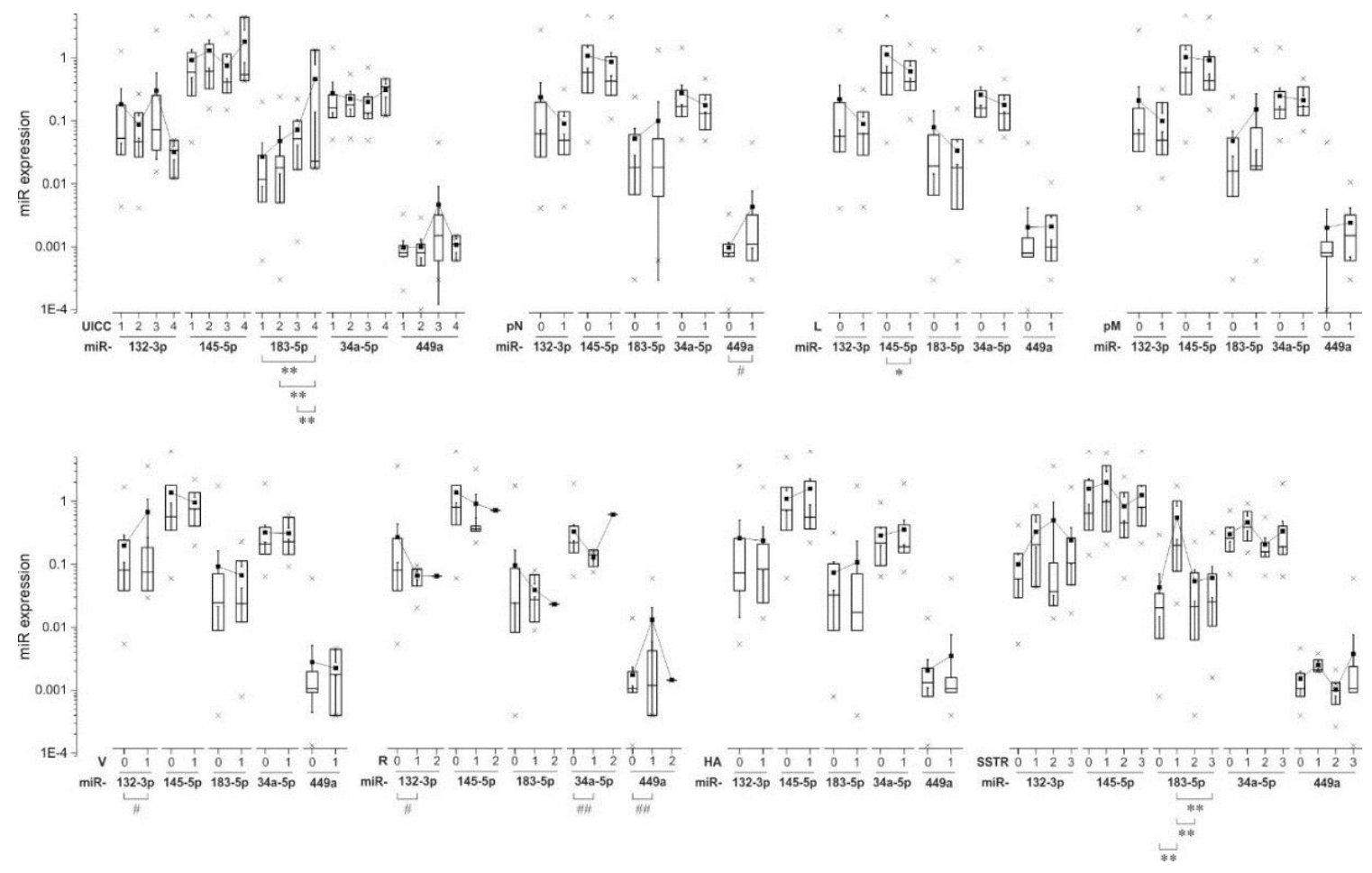

Figure 3. Relationship between miRNA expression and clinico-pathological data in pNET patients. Differences between expressions were tested using $t$-test or ANOVA and Bonferroni respectively. ${ }^{*} p<0.05,{ }^{* *} p<0.01$ - equal variances assumed, \#p<0.05, \#\# $p<0.01$-according to Levene variances not equal.

\subsection{Impact of miRNA Expression on DFS and OS}

The OS and DFS status-in this part of the analysis standing for the number of deceased patients (0-1) - was significantly linked to a higher expression of miRNA449a (equal variances assumed ${ }^{*}(0.012) /{ }^{*}(0.047)$ no homogeneity of variances (Levene) $(0.272,0.258) ; t$-test). To evaluate the miRNAs 132-3p, 145-5p, 183-5p, 34a-5p, and 449a as potential prognostic markers for DFS and OS we performed Kaplan-Meier survival analysis based on the relative miRNA expression levels using receiver operating characteristic (ROC) calculation with Youden Index analysis (Figure 4). We found a 
significant association between the expression of miRNA449a and OS (0.036, log-rank (Mantel-Cox)). Interestingly, evaluation of miRNA expression and DFS and OS revealed two groups: Expression of miRNAs 132-3p, 183-5p and 34a-5p was associated with higher OS and DFS values ("tumor suppressor miRNAs"), whereas the expression level of miRNA449a was associated with reduced survival rates ("oncogenic miRNA"). Surprisingly the expression of miRNA145-5p was associated with lower OS and enhanced DFS.
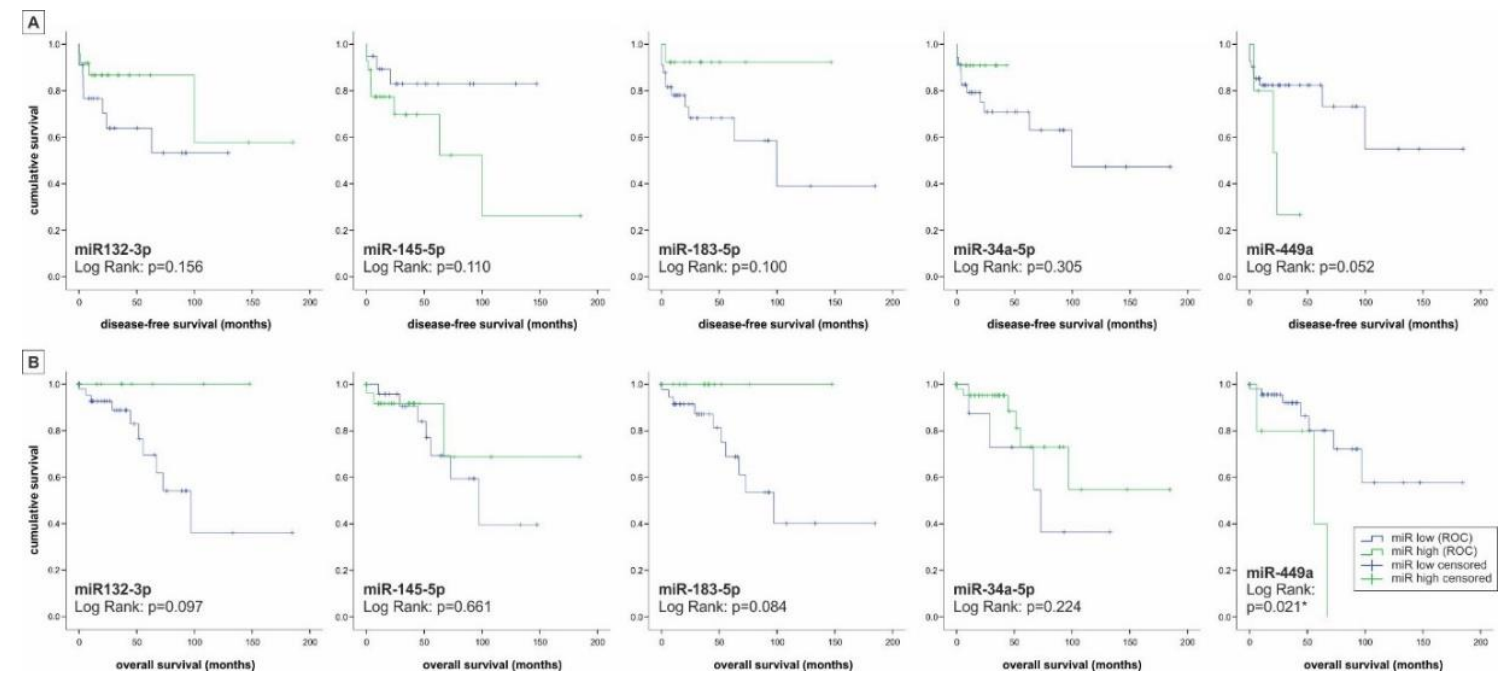

Figure 4. Survival analysis of patients with pNET. According to the classification of miRNAs in low or high based on the relative miRNA expression levels using receiver operating characteristic (ROC) calculation with Youden Index analysis the Kaplan-Meier survival analysis using the log-rank test was performed for (A) disease-free survival and (B) overall survival. "Protective miRNAs" include miRNA145-5p and miRNA183-5p, whereas "risk miRNAs" include miRNA132-3p, miRNA34a-5p, and miRNA449a. ${ }^{*} p<0.05,{ }^{* *} p<0.01$.

Finally, time-dependent multivariate analyses were applied to identify statistically significant predictors for OS and DFS including miRNA and HDAC scoring as well as clinico-pathological parameters (grading, TNM-staging, resection status, tumor size, hormone activity, localization). In the first step, the Cox-regression analysis of OS including all these mentioned parameters indicated only the $\mathrm{N}$-status (pN) (hazard ratio (HR): 692.3, 95\% confidence interval (CI): 0.103-4657763) as the strongest and highly significant predictor for OS ( $p<0.05$, log-rank (Mantel-Cox) test). Including only miRNAs, miRNA449a (HR: 4.6, 95\% CI: 1.1-19.6) alone and in binding with HDAC4 (cytoplasmatic) (HR: 1.02, 95\% CI: 1.005-1.035) could be filtered out as a significant predictor for OS ( $p<0.05$, log-rank (Mantel-Cox) test). In the next step, the Cox-regression analysis of DFS showed again only the N-status (hazard ratio (HR): 52.6, 95\% confidence interval (CI): 6.2-444.6) as the significant predictor for DFS in pNET-cases of all parameters ( $p<0.05, \log$-rank (Mantel-Cox) test). Interestingly, no miRNA could be detected as a significant prognostic parameter for DFS, whereby only the binding of HDAC4 (cytoplasmatic) and miRNA499a (HR: 1.011, 95\% CI: 0.999-1.024) reached significant levels $(p<0.05$, log-rank (Mantel-Cox) test).

\section{Discussion}

pNET is a relatively rare tumor entity with increasing incidence rates. Current therapeutic routes are limited, underlining the need for new therapeutic targets and treatment options. In the present immunohistochemical study we present initial information about the potential role of five "proliferation-associated" miRNAs in pNETs and their link with specific tumor-related HDACs. Hereby, we not only provide first data about a general involvement of these miRNAs in development and progression of pNETs, but also connect these miRNAs to HDAC expression and thus to 
epigenetic (de-)regulation. This is especially interesting since in a previous study investigating HDAC-deregulation in pNETs, we found specific HDAC family members to be linked with tumor grading, patient survival, and proliferation markers [22]. The expression levels of the set of miRNAs within our cohort of pNET samples followed a descending order with miRNA145-5p being expressed the highest and miRNA449a, the lowest (expression levels in descending order: 145-5p, 34a-5p, 132-3p, 183-5p, 449a). Micro-RNA145-5p does have tumor suppressor function as analyzed in multiple types of cancer like bladder cancer, renal cell carcinoma, lung cancer, esophageal squamous cell carcinoma, and breast cancer, which is particularly interesting as it showed the highest expression levels in our samples [23-28]. In our cohort, the miRNA with the lowest expression levels-miRNA-449-is also very well cited in connection with multiple tumor entities except pNETs. According to several studies focusing on this miRNA, it has a role as a tumor suppressor and is downregulated in hepatocellular carcinoma, lung cancer, gastric cancer, prostate cancer and also pancreatic cancer [19,29-32]. Two studies found a correlation between miRNA449 down-regulation and HDAC1/HDAC3 up-regulation; two HDAC family members important for tumor cell proliferation, [19,29]. In our study the expression of miRNA449a is significantly positively correlated with HDAC3/HDAC4- expression. Of note, there are reports suggesting an oncogenic role also for HDAC4 (repression of the cell cycle inhibitor p21) [33]. Accordingly, the correlation between miRNA449a and HDAC3/4 seems to be of an up-regulating nature in our pNET cohort. Interestingly, miRNA449a is significantly positively correlated with mitotic and proliferative activity in our pNET sample cohort even though its main function in other tumor entities throughout the literature is tumor suppressive and cell growth inhibiting [34]. Our observation that miRNA449a expression is also correlated with lower survival rates and lymph node infiltration further suggests a pro-tumorigenic role of this particular miRNA in pNETs. When comparing low-grade (G1, G2) to high-grade (G3) pNET samples, we found that miRNA145-5p, miRNA183-5p, and miRNA449a were upregulated in high-grade compared to low-grade pNETs, whereby miRNA132-3p and miRNA34a-5p were downregulated in high-grade pNETs compared to low-grade pNETs. Micro-RNA132-3p is especially interesting as it harbors both, tumor-promoting as well as tumor-suppressing functions [35-38]. Regarding miRNA34a-5p, Bommer et al. demonstrated that this miRNA contributes to proper p53 function and that its inactivation is likely to be involved in cancer development, which is coherent with our findings in that miRNA34a-5p shows low expression in high-grade pNETs [39]. Our evaluations of OS and DFS in the pNET sample cohort revealed that besides miRNA449a, also miRNA183-5p, alone and interestingly, partially in binding with HDAC4 (for miRNA449a) is significantly associated with reduced OS and DFS in survival analysis with Cox Proportional-Hazards Model. This observation is interesting as not only miRNA449a could be established as a tumor suppressor in some cancer types but also miRNA183-5p, suggesting potential tumor-specific interactions and effects for these miRNA species. Studies analyzing the role of miRNA-183-5p in cancer have on the one hand revealed tumor suppressive functions, but on the other hand, miRNA183-5p seems to play a promoting role in EMT, cell migration and invasion [40,41]. In gallbladder cancer for example miRNA-183-5p is upregulated and involved in tumorigenesis according to a study by Gao et al. [42]. Similar findings were published regarding gastric cancer and esophageal squamous cell carcinoma [43-45]. However, there are also studies that describe miRNA183-5p as a tumor suppressor. For example, Yang et al. showed that miRNA-183 acts as a tumor suppressor in human non-small cell lung cancer [40]. Moreover, in an in vitro study with human pancreatic cancer cell lines, miRNA-183 showed an anti-EMT effect [46]. In our study, miRNA-183-5p expression is significantly upregulated in higher-grade pNET samples according to UICC standards but in contrast to that, it is also correlated with higher OS and DFS rates. Shi and coworkers investigated the expression of miRNA449 in breast cancer specimens and found its expression significantly associated with poor survival. In addition, they detected a pro-proliferative effect of miRNA449 on breast cancer cells in in vitro studies [47]. Similar observations were obtained by Wang et al: Treatment of MCF-7 breast cancer cells with a miRNA449a inhibitor led to a significantly reduction of cell proliferation and migration [48]. As there are no studies so far investigating the role of miRNA449a in 
pNETs and as there is evidence of other miRNAs not distinctly having either a tumor suppressive- or oncogenic function, the results of this study indicate, that miRNA449a does function as an oncogene in pNETs in an interplay with HDAC3 and HDAC4. To confirm this, underlying epigenetic mechanisms should be further investigated. Then miRNA449a could be established as a prognostic factor for poor survival in pNETs and it might even be an interesting potential therapeutic target in combination with HDAC3/4 inhibition.

\section{Materials and Methods}

\subsection{Clinical and Pathological Characterization of pNET Cases}

The cohort included $n=57$ pNET cases that were surgically resected between 1997 and 2015 and archived at the Institute of Pathology, Paracelsus Medical University, Salzburg, Austria. All cases were comprehensively characterized for clinico-pathological parameters including sex, age, tumor size, localization, type of surgery, tumor grading, and staging. All cases were evaluated for grading (counting hematoxylin-eosin mitosis in 10 consecutive high power fields (HPF) according to published guidelines [49]), neuroendocrine differentiation as well as mitotic, proliferative and hormonal activity by immunohistochemistry on $5 \mu \mathrm{m}$ formalin-fixed paraffin-embedded (FFPE) tissue sections as described in detail previously [50,51]. This study was conducted following the national and institutional guidelines of the Paracelsus Medical University Salzburg/Salzburg county hospital as well as in accordance with the declaration of Helsinki (1964); the anonymized samples are exclusively available for research purposes in retrospective studies. The analyses of human pNET samples were approved by the local ethics committee (415-EP/73/408-2014) (19 May 2014).

\subsection{Isolation of Total miRNA from FFPE Samples and Expression Analysis by RT-PCR}

Using a RM2245 semi-automated rotator microtome (Leica Biosystems Nussloch GmbH, Nussloch, Germany), one to three $10 \mu \mathrm{m}$ sections (depending on tumor size) were cut from FFPE blocks of tumor samples after microdissection to remove surrounding tissue. These sections were transferred to $1.5 \mathrm{~mL}$ tubes, and subsequently, miRNA isolation was performed using the miRNeasy FFPE Kit. For subsequent RT-PCR analysis, microRNAs were transcribed utilizing the miScript II RT ${ }^{\mathrm{TM}}$ kit. All FFPE miR reagents and qPCR kits were obtained from Qiagen (Hilden, Germany) and used according to the manufacturer's instructions.

\subsection{Immunohistochemistry and Processing for Markers of HDACs (1-6, 8-11 and Sirt1)}

To simplify the investigations and ensure comparability of the immunohistochemical signals, five tissue microarrays (TMA) covering all 57 cases were assembled. If possible, each pNET case was represented on the TMA with tumor tissue as well as adjacent healthy pancreatic tissue [52]. Each TMA was cut into $5 \mu \mathrm{m}$ sections, raised on adhesive glass slides and dried at $60{ }^{\circ} \mathrm{C}$ for one hour. Deparaffinization, antigen retrieval, immunostaining, counterstaining, dehydration, and coverslip application, as well as pre-treatment, were performed using standardized routine immunohistochemistry (IHC) protocols. Immunohistochemical staining was performed on a Dako Autostainer Plus combined with the EnVision Plus System using primary mouse or rabbit antibodies (Dako, Vienna, Austria). In case of a primary goat antibody, a secondary enzyme conjugated antibody was used. Sources of all applied primary antibodies (including catalog-number, clone species, dilution, incubation, and pretreatment) are summarized in Supplementary Table S1.

\subsection{Interpretation and Scoring of the IHC}

Examination of each pNET case within the TMAs was carried out semi-quantitatively by counting the number of stained cells and assessing the intensity of the staining. According to the data sheets of the chosen HDAC antibodies, a cytoplasmatic (cyt: only HDAC 4, 5, 8, and 10) and/or a nuclear (nuc: all HDACs) expression pattern was separately evaluated. Afterwards an immunoreactivity 
score (range 0-300) was calculated by multiplying the scores for intensity (0-3) and the stained cells (extensity, $0-100 \%$ ) as previously published [53]. In addition, absolute quantitative scoring data of each HDAC, the relative data of low and high HDAC expressions levels were estimated according to the expression's means as HDAC-specific cut-off-values, as previously published [22].

\subsection{Statistical Analysis}

Statistical analysis was performed with SPSS 24.0 (IBM Corporation, New York, USA). The $\chi^{2}$-test (nominal), Student's $t$-test (interval) and univariate ANOVA (analysis of variance, Bonferroni post-hoc test) were used to analyze differences between two and more groups of tissue samples, respectively. Pearson's correlation coefficient was applied for correlation analysis. Cut-off-values for summary variables were determined using the receiver operating characteristic (ROC) calculation and Youden Index analysis for disease-free and overall survival (DFS, OS). For survival analysis, cases with missing date of death were excluded. Univariate survival analysis was performed using the Kaplan-Meier method comparing the survival curves with the log-rank test. For multivariate analysis, the time-dependent Cox proportional hazards model was performed to identify independent predictors for DFS and OS using the forward elimination Wald method. For all calculations, $p<0.05$ and $p<0.01$ were considered as significant or highly significant, respectively.

\section{Conclusions}

Taken together, our study shows that two members of the HDAC family are linked with miRNA expression in pNETs. The most interesting association is between miRNA449a and HDAC3 and HDAC4. According to our data regarding clinico-pathological, proliferative, mitotic characteristics and the survival analysis, it seems that in pNETs miRNA449a does have an oncogenic function as opposed to in other tumor entities.

Supplementary Materials: Supplementary materials can be found at http:/ / www.mdpi.com/1422-0067/19/9/ $2781 /$ s1

Author Contributions: Conceptualization, D.N. and K.H.; Methodology, E.K., R.U., S.Swierczynski and T.K.; Software, D.N. and T.K.; Validation, D.N. and K.H.; Formal Analysis, D.N. and K.H.; Investigation, E.K., R.U., S.Swierczynski and T.K.; Writing-Original Draft Preparation, K.H.; Writing-Review \& Editing, S.Stättner, F.P., T.J., C.M., T.K., P.D.F. and D.N.; Visualization, T.K., D.N. and K.H.; Supervision, D.N.; Project Administration, D.N.; Funding Acquisition, D.N.

Funding: This work was supported by the Wissenschaftlicher Verein der Pathologie Salzburg/Austria and by an "unrestricted research grant/other research grant" of Novartis Pharma GmbH/AUSTRIA.

Acknowledgments: The expert technical assistance of Berta Lechner and Monika Prinz as well as Brian van Merkestijn is gratefully acknowledged.

Conflicts of Interest: The authors declare no conflict of interest.

$\begin{array}{ll}\text { Abbreviations } \\ \text { Cyt } & \text { Cytoplasmatic } \\ \text { DFS } & \text { Disease-free survival } \\ \text { EMT } & \text { Epithelial to mesenchymal transition } \\ \text { FFPE } & \text { Formalin-fixed paraffin-embedded } \\ \text { HDAC } & \text { Histone deacetylases } \\ \text { HPF } & \text { High power fields } \\ \text { IHC } & \text { Immunohistochemistry } \\ \text { miRNA } & \text { microRNA } \\ \text { nuc/ncl } & \text { Nuclear } \\ \text { OS } & \text { Overall survival } \\ \text { pNET } & \text { Pancreatic neuroendocrine tumor } \\ \text { SD } & \text { Standard deviation } \\ \text { TMA } & \text { Tissue microarray }\end{array}$




\section{References}

1. Alexandraki, K.I.; Kaltsas, G. Gastroenteropancreatic neuroendocrine tumors: New insights in the diagnosis and therapy. Endocrine 2012, 41, 40-52. [CrossRef] [PubMed]

2. Schimmack, S.; Svejda, B.; Lawrence, B.; Kidd, M.; Modlin, I.M. The diversity and commonalities of gastroenteropancreatic neuroendocrine tumors. Langenbecks Arch. Surg. 2011, 396, 273-298. [CrossRef] [PubMed]

3. Lawrence, B.; Gustafsson, B.I.; Chan, A.; Svejda, B.; Kidd, M.; Modlin, I.M. The epidemiology of gastroenteropancreatic neuroendocrine tumors. Endocrinol. Metab. Clin. N. Am. 2011, 40, 1-18. [CrossRef] [PubMed]

4. Ehehalt, F.; Saeger, H.D.; Schmidt, C.M.; Grutzmann, R. Neuroendocrine tumors of the pancreas. Oncologist 2009, 14, 456-467. [CrossRef] [PubMed]

5. Alexandraki, K.I.; Karapanagioti, A.; Karoumpalis, I.; Boutzios, G.; Kaltsas, G.A. Advances and current concepts in the medical management of gastroenteropancreatic neuroendocrine neoplasms. BioMed Res. Int. 2017, 2017, 9856140. [CrossRef] [PubMed]

6. Virani, S.; Colacino, J.A.; Kim, J.H.; Rozek, L.S. Cancer epigenetics: A brief review. ILAR J. 2012, 53, 359-369. [CrossRef] [PubMed]

7. Kanwal, R.; Gupta, K.; Gupta, S. Cancer epigenetics: An introduction. Methods Mol. Biol. 2015, 1238, 3-25. [PubMed]

8. Felsenfeld, G. A brief history of epigenetics. Cold Spring Harb. Perspect. Biol. 2014, 6, a018200. [CrossRef] [PubMed]

9. He, L.; Hannon, G.J. MicroRNAs: Small RNAs with a big role in gene regulation. Nat. Rev. Genet. 2004, 5, 522-531. [CrossRef] [PubMed]

10. Ropero, S.; Esteller, M. The role of histone deacetylases (HDACs) in human cancer. Mol. Oncol. 2007, 1, 19-25. [CrossRef] [PubMed]

11. Guo, M.; Jia, Y.; Yu, Z.; House, M.G.; Esteller, M.; Brock, M.V.; Herman, J.G. Epigenetic changes associated with neoplasms of the exocrine and endocrine pancreas. Discov. Med. 2014, 17, 67-73. [PubMed]

12. Klieser, E.; Swierczynski, S.; Mayr, C.; Schmidt, J.; Neureiter, D.; Kiesslich, T.; Illig, R. Role of histone deacetylases in pancreas: Implications for pathogenesis and therapy. World J. Gastrointest. Oncol. 2015, 7, 473-483. [CrossRef] [PubMed]

13. Croce, C.M. Causes and consequences of microRNA dysregulation in cancer. Nat. Rev. Genet. 2009, 10, 704-714. [CrossRef] [PubMed]

14. Fiaschetti, G.; Abela, L.; Nonoguchi, N.; Dubuc, A.M.; Remke, M.; Boro, A.; Grunder, E.; Siler, U.; Ohgaki, H.; Taylor, M.D.; et al. Epigenetic silencing of miRNA-9 is associated with HES1 oncogenic activity and poor prognosis of medulloblastoma. Br. J. Cancer 2014, 110, 636-647. [CrossRef] [PubMed]

15. Holubekova, V.; Mendelova, A.; Jasek, K.; Mersakova, S.; Zubor, P.; Lasabova, Z. Epigenetic regulation by DNA methylation and miRNA molecules in cancer. Future Oncol. 2017, 13, 2217-2222. [CrossRef] [PubMed]

16. Kwon, H.; Song, K.; Han, C.; Zhang, J.; Lu, L.; Chen, W.; Wu, T. Epigenetic silencing of miRNA-34a in human cholangiocarcinoma via EZH2 and DNA methylation: Impact on regulation of notch pathway. Am. J. Pathol. 2017, 187, 2288-2299. [CrossRef] [PubMed]

17. Li, Y.; Xu, Z.; Li, B.; Zhang, Z.; Luo, H.; Wang, Y.; Lu, Z.; Wu, X. Epigenetic silencing of miRNA-9 is correlated with promoter-proximal $\mathrm{CpG}$ island hypermethylation in gastric cancer in vitro and in vivo. Int. J. Oncol. 2014, 45, 2576-2586. [CrossRef] [PubMed]

18. Mayr, C.; Beyreis, M.; Wagner, A.; Pichler, M.; Neureiter, D.; Kiesslich, T. Deregulated microRNAs in biliary tract cancer: Functional targets and potential biomarkers. BioMed Res. Int. 2016, 2016, 4805270. [CrossRef] [PubMed]

19. Noonan, E.J.; Place, R.F.; Pookot, D.; Basak, S.; Whitson, J.M.; Hirata, H.; Giardina, C.; Dahiya, R. miR-449a targets HDAC-1 and induces growth arrest in prostate cancer. Oncogene 2009, 28, 1714-1724. [CrossRef] [PubMed]

20. Sampath, D.; Liu, C.; Vasan, K.; Sulda, M.; Puduvalli, V.K.; Wierda, W.G.; Keating, M.J. Histone deacetylases mediate the silencing of miR-15a, miR-16, and miR-29b in chronic lymphocytic leukemia. Blood 2012, 119, 1162-1172. [CrossRef] [PubMed] 
21. Swierczynski, S.; Klieser, E.; Illig, R.; Alinger-Scharinger, B.; Kiesslich, T.; Neureiter, D. Histone deacetylation meets miRNA: Epigenetics and post-transcriptional regulation in cancer and chronic diseases. Expert Opin. Biol. Ther. 2015, 15, 651-664. [CrossRef] [PubMed]

22. Klieser, E.; Urbas, R.; Stattner, S.; Primavesi, F.; Jager, T.; Dinnewitzer, A.; Mayr, C.; Kiesslich, T.; Holzmann, K.; Di Fazio, P.; et al. Comprehensive immunohistochemical analysis of histone deacetylases in pancreatic neuroendocrine tumors: HDAC5 as a predictor of poor clinical outcome. Hum. Pathol. 2017, 65, 41-52. [CrossRef] [PubMed]

23. Lu, Y.; Govindan, R.; Wang, L.; Liu, P.Y.; Goodgame, B.; Wen, W.; Sezhiyan, A.; Pfeifer, J.; Li, Y.F.; Hua, X.; et al. MicroRNA profiling and prediction of recurrence/relapse-free survival in stage I lung cancer. Carcinogenesis 2012, 33, 1046-1054. [CrossRef] [PubMed]

24. Han, Q.; Zhang, H.Y.; Zhong, B.L.; Wang, X.J.; Zhang, B.; Chen, H. MicroRNA-145 inhibits cell migration and invasion and regulates epithelial-mesenchymal transition (EMT) by targeting connective tissue growth factor (CTGF) in esophageal squamous cell carcinoma. Med. Sci. Monit. 2016, 22, 3925-3934. [CrossRef] [PubMed]

25. Doberstein, K.; Steinmeyer, N.; Hartmetz, A.K.; Eberhardt, W.; Mittelbronn, M.; Harter, P.N.; Juengel, E.; Blaheta, R.; Pfeilschifter, J.; Gutwein, P. MicroRNA-145 targets the metalloprotease ADAM17 and is suppressed in renal cell carcinoma patients. Neoplasia 2013, 15, 218-230. [CrossRef] [PubMed]

26. Larne, O.; Hagman, Z.; Lilja, H.; Bjartell, A.; Edsjo, A.; Ceder, Y. MiR-145 suppress the androgen receptor in prostate cancer cells and correlates to prostate cancer prognosis. Carcinogenesis 2015, 36, 858-866. [CrossRef] [PubMed]

27. Chiyomaru, T.; Enokida, H.; Tatarano, S.; Kawahara, K.; Uchida, Y.; Nishiyama, K.; Fujimura, L.; Kikkawa, N.; Seki, N.; Nakagawa, M. MiR-145 and miR-133a function as tumour suppressors and directly regulate FSCN1 expression in bladder cancer. Br. J. Cancer 2010, 102, 883-891. [CrossRef] [PubMed]

28. Wu, J.; Yin, L.; Jiang, N.; Guo, W.J.; Gu, J.J.; Chen, M.; Xia, Y.Y.; Wu, J.Z.; Chen, D.; Wu, J.F.; et al. MiR-145, a microRNA targeting ADAM17, inhibits the invasion and migration of nasopharyngeal carcinoma cells. Exp. Cell Res. 2015, 338, 232-238. [CrossRef] [PubMed]

29. Buurman, R.; Gurlevik, E.; Schaffer, V.; Eilers, M.; Sandbothe, M.; Kreipe, H.; Wilkens, L.; Schlegelberger, B.; Kuhnel, F.; Skawran, B. Histone deacetylases activate hepatocyte growth factor signaling by repressing microRNA-449 in hepatocellular carcinoma cells. Gastroenterology 2012, 143, 811-820. [CrossRef] [PubMed]

30. Jeon, H.S.; Lee, S.Y.; Lee, E.J.; Yun, S.C.; Cha, E.J.; Choi, E.; Na, M.J.; Park, J.Y.; Kang, J.; Son, J.W. Combining microRNA-449a/b with a HDAC inhibitor has a synergistic effect on growth arrest in lung cancer. Lung Cancer 2012, 76, 171-176. [CrossRef] [PubMed]

31. Li, F.; Liang, J.; Bai, L. MicroRNA-449a functions as a tumor suppressor in pancreatic cancer by the epigenetic regulation of ATDC expression. Biomed. Pharmacother. 2018, 103, 782-789. [CrossRef] [PubMed]

32. Bou Kheir, T.; Futoma-Kazmierczak, E.; Jacobsen, A.; Krogh, A.; Bardram, L.; Hother, C.; Gronbaek, K.; Federspiel, B.; Lund, A.H.; Friis-Hansen, L. MiR-449 inhibits cell proliferation and is down-regulated in gastric cancer. Mol. Cancer 2011, 10, 29. [CrossRef] [PubMed]

33. Kang, Z.H.; Wang, C.Y.; Zhang, W.L.; Zhang, J.T.; Yuan, C.H.; Zhao, P.W.; Lin, Y.Y.; Hong, S.; Li, C.Y.; Wang, L. Histone deacetylase HDAC4 promotes gastric cancer SGC-7901 cells progression via p21 repression. PLOS ONE 2014, 9, e98894. [CrossRef] [PubMed]

34. Yong-Ming, H.; Ai-Jun, J.; Xiao-Yue, X.; Jian-Wei, L.; Chen, Y.; Ye, C. miR-449a: A potential therapeutic agent for cancer. Anti-Cancer Drugs 2017, 28, 1067-1078. [CrossRef] [PubMed]

35. Yang, L.; Belaguli, N.; Berger, D.H. MicroRNA and colorectal cancer. World J. Surg. 2009, 33, 638-646. [CrossRef] [PubMed]

36. Anand, S.; Majeti, B.K.; Acevedo, L.M.; Murphy, E.A.; Mukthavaram, R.; Scheppke, L.; Huang, M.; Shields, D.J.; Lindquist, J.N.; Lapinski, P.E.; et al. MicroRNA-132-mediated loss of p120RasGAP activates the endothelium to facilitate pathological angiogenesis. Nat. Med. 2010, 16, 909-914. [CrossRef] [PubMed]

37. Formosa, A.; Lena, A.M.; Markert, E.K.; Cortelli, S.; Miano, R.; Mauriello, A.; Croce, N.; Vandesompele, J.; Mestdagh, P.; Finazzi-Agro, E.; et al. DNA methylation silences miR-132 in prostate cancer. Oncogene 2013, 32, 127-134. [CrossRef] [PubMed]

38. Yang, J.; Gao, T.; Tang, J.; Cai, H.; Lin, L.; Fu, S. Loss of microRNA-132 predicts poor prognosis in patients with primary osteosarcoma. Mol. Cell. Biochem. 2013, 381, 9-15. [CrossRef] [PubMed] 
39. Bommer, G.T.; Gerin, I.; Feng, Y.; Kaczorowski, A.J.; Kuick, R.; Love, R.E.; Zhai, Y.; Giordano, T.J.; Qin, Z.S.; Moore, B.B.; et al. p53-mediated activation of miRNA34 candidate tumor-suppressor genes. Curr. Biol. 2007, 17, 1298-1307. [CrossRef] [PubMed]

40. Yang, C.L.; Zheng, X.L.; Ye, K.; Ge, H.; Sun, Y.N.; Lu, Y.F.; Fan, Q.X. MicroRNA-183 acts as a tumor suppressor in human non-small cell lung cancer by down-regulating MTA1. Cell. Physiol. Biochem. 2018, 46, 93-106. [CrossRef] [PubMed]

41. Xiong, H.; Chen, R.; Liu, S.; Lin, Q.; Chen, H.; Jiang, Q. MicroRNA-183 induces epithelial-mesenchymal transition and promotes endometrial cancer cell migration and invasion in by targeting CPEB1. J. Cell. Biochem. 2018. [CrossRef] [PubMed]

42. Gao, J.M.; Huang, L.Z.; Huang, Z.G.; He, R.Q. Clinical value and potential pathways of miR-183-5p in bladder cancer: A study based on miRNA-seq data and bioinformatics analysis. Oncol. Lett. 2018, 15, 5056-5070. [CrossRef] [PubMed]

43. Gu, W.; Gao, T.; Shen, J.; Sun, Y.; Zheng, X.; Wang, J.; Ma, J.; Hu, X.Y.; Li, J.; Hu, M.J. MicroRNA-183 inhibits apoptosis and promotes proliferation and invasion of gastric cancer cells by targeting PDCD4. Int. J. Clin. Exp. Med. 2014, 7, 2519-2529. [PubMed]

44. Ren, L.H.; Chen, W.X.; Li, S.; He, X.Y.; Zhang, Z.M.; Li, M.; Cao, R.S.; Hao, B.; Zhang, H.J.; Qiu, H.Q.; et al. MicroRNA-183 promotes proliferation and invasion in oesophageal squamous cell carcinoma by targeting programmed cell death 4. Br. J. Cancer 2014, 111, 2003-2013. [CrossRef] [PubMed]

45. Yang, M.; Liu, R.; Li, X.; Liao, J.; Pu, Y.; Pan, E.; Yin, L.; Wang, Y. miRNA-183 suppresses apoptosis and promotes proliferation in esophageal cancer by targeting PDCD4. Mol. Cell. 2014, 37, 873-880. [CrossRef] [PubMed]

46. Lin, X.; Zheng, L.; Song, H.; Xiao, J.; Pan, B.; Chen, H.; Jin, X.; Yu, H. Effects of microRNA-183 on epithelial-mesenchymal transition, proliferation, migration, invasion and apoptosis in human pancreatic cancer SW1900 cells by targeting MTA1. Exp. Mol. Pathol. 2017, 102, 522-532. [CrossRef] [PubMed]

47. Shi, W.; Bruce, J.; Lee, M.; Yue, S.; Rowe, M.; Pintilie, M.; Kogo, R.; Bissey, P.A.; Fyles, A.; Yip, K.W.; et al. MiR-449a promotes breast cancer progression by targeting CRIP2. Oncotarget 2016, 7, 18906-18918. [CrossRef] [PubMed]

48. Wang, H.L.; Xiao, Y.; Wu, L.; Ma, D.C. Effects of miR-449a on proliferation and migration of human breast cancer cell line MCF-7. Zhongguo Ying Yong Sheng Li Xue Za Zhi 2017, 33, 508-513. (In Chinese) [PubMed]

49. Bosman, F.T.; Carneiro, F.; Hruban, R.H.; Theise, N.D. WHO Classification of Tumours of the Digestive System, 4th ed.; World Health Organization: Geneva, Switzerland, 2010.

50. Kemmerling, R.; Weyland, D.; Kiesslich, T.; Illig, R.; Klieser, E.; Jager, T.; Dietze, O.; Neureiter, D. Robust linear regression model of Ki-67 for mitotic rate in gastrointestinal stromal tumors. Oncol. Lett. 2014, 7, 745-749. [CrossRef] [PubMed]

51. Klieser, E.; Illig, R.; Stattner, S.; Primavesi, F.; Jager, T.; Swierczynski, S.; Kiesslich, T.; Kemmerling, R.; Bollmann, C.; Di Fazio, P.; et al. Endoplasmic reticulum stress in pancreatic neuroendocrine tumors is linked to clinicopathological parameters and possible epigenetic regulations. Anticancer Res. 2015, 35, 6127-6136. [PubMed]

52. Kemmerling, R.; Alinger, B.; Dietze, O.; Bosmuller, H.C.; Ocker, M.; Wolkersdorfer, G.W.; Berr, F.; Neureiter, D.; Kiesslich, T. Association of stem cell marker expression pattern and survival in human biliary tract cancer. Int. J. Oncol. 2012, 41, 511-522. [CrossRef] [PubMed]

53. Kiesslich, T.; Alinger, B.; Wolkersdorfer, G.W.; Ocker, M.; Neureiter, D.; Berr, F. Active Wnt signalling is associated with low differentiation and high proliferation in human biliary tract cancer in vitro and in vivo and is sensitive to pharmacological inhibition. Int. J. Oncol. 2010, 36, 49-58. [PubMed]

(C) 2018 by the authors. Licensee MDPI, Basel, Switzerland. This article is an open access article distributed under the terms and conditions of the Creative Commons Attribution (CC BY) license (http:/ / creativecommons.org/licenses/by/4.0/). 